\title{
High Expression of MDR1 is Correlated with Poor Prognosis for Common Malignancies
}

\author{
Bo Gao ${ }^{1,2}$, Fengmei Yang 3 , Wei Chen ${ }^{2}$, Xiaojia Song ${ }^{4}$, Xiaobo $\mathrm{Liu}^{5}$ and Dongmin $\mathrm{Li}^{1 *}$ \\ ${ }^{1}$ Department of Biochemistry and Molecular Biology, School of Basic Medical Sciences, \\ Xi'an Jiaotong University Health Science Center, Xi'an, Shanxi 710061, P.R. China. \\ ${ }^{2}$ Department of Laboratory Medicine, Taihe Hospital, Hubei University of Medicine, \\ Shiyan, Hubei 442000, P.R. China. \\ ${ }^{3}$ Department of Obstetrics and Gynecology, Taihe Hospital, Hubei University of \\ Medicine, Shiyan, Hubei 442000, P.R. China. \\ ${ }^{4}$ Shiyan Prefecture Center for Disease Control and Prevention, Shiyan, Hubei 442000, \\ P.R. China. \\ ${ }^{5}$ Department of Gastroenterology, Taihe Hospital, Hubei University of Medicine, Shiyan, \\ Hubei 442000, P.R. China.
}

Bo Gao and Fengmei Yang contributed equally.

\begin{abstract}
A B S T R A C T
The purpose of this study is to detect the relative expression level of Multi-drug resistance gene (MDR1) in common tumor malignancies, and evaluate the prognostic value of MDR1 expression in common malignancies. mRNA relative expression levels of MDR1 were detected byreal-time quantitative polymerase chain reaction (RT-qPCR) in tumor, adjacent, and non-cancerous tissues. The tumor markers were detected with COBAS 6000 . The prognostic value of relative MDR1 expression level in malignant tumors was investigated by univariate survival and Cox regression model analyses, and survival times were compared using the log-rank test. At the same time, through receiver operating characteristic (ROC) curve analysis, their diagnostic threshold values were calculated. MDR1 expression levels were the highest in malignant tumor tissues, followed by adjacent tissues, and the lowest in non-cancerous tissues. Differences in expression level between varying degrees of differentiation and/or lymphatic metastasis, as well as variations in negative and positive expression between survival and recurrence times, were statistically significant. The level of tumor markers at 6 months after operation was significantly lower than that before operation in recurrent/non-recurrent and MDR1 positive/MDR1 negative group. There was a significant correlation between MDR1 expression and tumor markers (CA125 and CA153), regardless of whether recurrence was involved in PEOC and breast cancer. Multivariate logistic regression indicated that relative MDR1 expression levels in patients of the positive-group survival curves were lower than those in patients of the negative-group curves. High MDR1 expression is associated with clinicopathological features in malignant tumor patients. The detection of MDR1 expression combined with tumor markers can improve the sensitivity and specificity of predicting postoperative recurrence (especially breast cancer and PEOC).
\end{abstract}

\begin{tabular}{l} 
Article Information \\
\hline Received 02 August 2019 \\
Revised 30 August 2019 \\
Accepted 07 September 2019 \\
Available online 06 April 2020 \\
Authors' Contribution \\
\hline BG and DL conceived and designed \\
the study. FY and WC performed the \\
experiments. XL and XL analyzed the \\
data. BG wrote the manuscript. \\
Key words \\
\hline MDR1, Malignant tumor, Prognosis, \\
ROC, Expression
\end{tabular}

\section{INTRODUCTION}

$\mathrm{T}$ umors threaten human health and present a significant burden to families. In China, the incidence and mortality of common malignant tumors is high (Chen et al., 2016). At present, tumor drug therapy via chemotherapy and targeted therapy is the most widely used antitumor measure. Chemotherapy failure in most patients is caused by Multi-drug resistance (MDR) induced by

\footnotetext{
* Corresponding author: lidongm@mail.xjtu.edu.cn 0030-9923/2020/0004-1501 \$9.00/0

Copyright 2020 Zoological Society of Pakistan
}

chemotherapeutic drugs (Abraham et al., 2015). In MDR, tumor cells are not only resistant to antitumor drugs but also cross-resistant to other drugs with different structures and mechanisms. There are two types of MDR, the first is acquired resistance, in which some tumors have good early-treatment effects and poor efficacy in later stages, and the second is primary resistance, in which drug resistance is developed at the beginning of chemotherapy (Joshi et al., 2017). Overcoming the poor curative effects caused by MDR is one of the main directions of oncology research.

MDR1 encodes the drug transporter P-glycoprotein (P-gp), which is the earliest known drug resistance gene/ protein. P-gp is considered a symbol of MDR because 
the mechanism it uses to regulate drug resistance is the classical mechanism of MDR. P-gp releases ATP to pump drugs and metabolic poisons out of the cell to reduce intracellular concentrations, thereby protecting cells from damage. This mechanism is primarily used by tumor cells to generate MDR (Yan et al., 2014). P-gP was mainly resistant to vinblastine and doxorubicin, but increased MDR expression can lead to accumulation of intracellular chemotherapeutic drugs, decreased drug sensitivity, and increased formation rates of MDR-derived malignant tumors (Yan et al., 2014), such as colorectal carcinoma (Liu et al., 2014), lung cancer (Zhao et al., 2018), gastric cancer (Mieszala et al., 2018) and breast cancer (Ge et al., 2017), so it was of great clinical significance for tumor patients to choose chemotherapy regimen and prognosis. In this paper, the expression of MDR1/P-gp in common malignant tumors and the role of this expression in MDR tumors were studied. The relationship between expression and clinic pathological features and the value of MDR1/Pgp in predicting tumor prognosis were also analyzed.

\section{MATERIALS AND METHODS}

\section{Specimens and patients}

Different types of fresh specimens were collected from May 2008 to August 2011 in Taihe Hospital. All specimens were collected including cancer tissues, adjacent tissues ( $>2 \mathrm{~cm}$ distance to the resection margin) and non-cancerous ( $>5 \mathrm{~cm}$ distance to the resection margin), some patients did not collect adjacent tissues or non-cancerous. All patients were collected with relevant case data, including gastric cancer (gender, size, pathogenetic location, lymph node status, histologic type), colorectal cancer (gender, age, size, lymph node status, differentiation, Duke stage), primary epithelial ovarian cancer (age, histologic type, stage, differentiation), non-small cell lung cancer (gender, age, histologic type, stage, differentiation, lymph node status), breast cancer (age, histologic type, TNM stage, menopausal status). There were not statistically significant with the demographic and clinical information in all cases. According to the imaging and laboratory examination, the possibility of postoperative recurrence of malignant tumor can be determined. None of the patients underwent preoperative radiotherapy or chemotherapy. All patients had complete clinical date, and were followed up from 6 to 60 months. In accordance with the Declaration of Helsinki, all protocols were approved by the Ethics Committee of Taihe Hospital.

Detection of tumor markers in common tumor malignancies Peripheral blood $5 \mathrm{ml}$ was collected pre-operation into a heparin anticoagulant tube for the isolation of plasma.
The tumor markers were detected with COBAS 6000 (Roche) at two time points, in one week of post-operation and 6 months after the surgery, with CEA $>10 \mu \mathrm{g} / \mathrm{L}$, CA $724>6 \mathrm{KU} / \mathrm{L}, \mathrm{CA} 153>25 \mathrm{KU} / \mathrm{L}, \mathrm{CA} 125>35 \mathrm{KU} / \mathrm{L}$, and CYFRA $21-1>3.3 \mathrm{ng} / \mathrm{mL}$ being regarded as elevated status.

\section{Real-time PCR analysis}

Total RNA of Tissues and cells were extracted using TRIzol ${ }^{\circledR}$ Reagent according to the instruction. The absorbance of RNA was determined at $260 \mathrm{~nm}$ and $280 \mathrm{~nm}$ with Nano Drop-2000. cDNA was synthesized according to the instruction by the Reverse Transcription System kit and was stored at $-80^{\circ} \mathrm{C}$ until use. The primers were used for Realtime quantitative PCR (RT-qPCR) as follows: Forward, 5'-CCC ATC ATT GCA ATA GCA GG-3' and reverse, 5'-TGT TCA AAC TTC TGC TCC TGA-3' for human MDR1; and forward, 5'-GAA GGT GAA GGT CGG AGT C-3' and reverse, 5'- GAA GAT GGT GAT GGG ATT TC-3' for human GAPDH. MDR1, GAPDH primers yielded products of $158,226 \mathrm{bp}$, respectively. PCR amplification system: $\mathrm{Mg}^{2+} 2.4 \mu \mathrm{l}, 5$ ' and 3' primer $2 \mu \mathrm{l}, 2 \mathrm{mmol} / \mathrm{L}$ dNTP $1.5 \mu \mathrm{l}, 10 \times \mathrm{SYBR}-G r e e n$ $1 \mu \mathrm{l}$, Taq $0.3 \mu 1,10 \times$ Buffer $3 \mu \mathrm{l}$, cDNA5 $\mu \mathrm{l}$, with sterile water total volume filled $30 \mu \mathrm{l}$. Reaction conditions: $95^{\circ} \mathrm{C}$ denaturation for 5 minutes, $94^{\circ} \mathrm{C} 30$ seconds, $60^{\circ} \mathrm{C} 30$ seconds, $72^{\circ} \mathrm{C} 1$ min with 35 cycles, and dissociation curve analysis was performed after all amplification. MDR1 gene relative expression level was normalized by GAPDH in each sample and determined by the $2-\Delta^{\Delta \mathrm{Ct}}$ method.

\section{Statistical analysis}

Statistical analysis was performed with SPSS software version 16.0 and $P<0.05$ was used to indicate statistically significant difference. The measured data were expressed as the mean \pm standard deviation. Survival time analysis was compared using the log rank test. A Student's t-test was used to determine the expression differences between the different groups, Univariate survival analysis and Cox regression model analysis were used to compare different clinical feature groups. Receiver operating characteristic (ROC) curve was established to evaluate the recurrent value of MDR1 and tumor markers in common malignant tumor. Partial correlations were used to analyze the correlation between MDR1 expression and tumor markers' levels.

\section{RESULTS}

\section{MDRl gene relative expression levels in all samples}

MDR1 was highly expressed in all malignant tumor tissues, partially expressed in adjacent tissues, and almost not expressed in non-cancerous tissues, the difference of expression between different groups were statistically 
significant (Fig. 1). Statistical analysis found that the differences in MDR1 expression levels of differentiation degree/lymph node status were statistically significant (Table I).

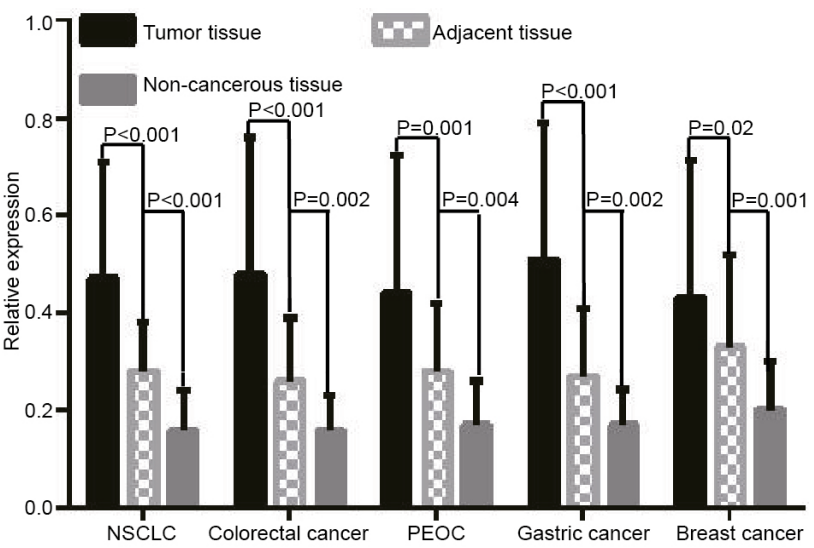

Fig. 1. The relative expression levels of MDR1 in all tissues.

\section{Recurrence time and survival time in different groups}

Most patients had relapses during the follow-up period, and the recurrence rate of non-small cell lung cancer was $53.2 \%(25 / 47)$, gastric cancer $30.2 \%(16 / 53)$, primary epithelial ovarian cancer was 73.7\% (42/57), colorectal carcinoma was $56.9 \%$ (29/51), breast cancer was $29.2 \%$ (14/48). According to the expression of resistance gene, it was divided into positive and negative group. The survival time and recurrence time of the follow-up patients were shown in Figure 2.

\section{The levels of different tumor markers in different groups}

In the malignant tumors, the tumor markers were reduced in both the recurrence group/the non-recurrence group and MDR1 positive group/MDR1 negative group, the difference was statistically significant $(P<0.001)$. Before the operation, there was no statistically significant difference in the level of tumor markers between the recurrence group/the non-recurrence group and MDR1 positive group/MDR1 negative group. But after surgery, there was statistically significant difference in the level of tumor markers between the recurrence group/the non-recurrence group and MDR1 positive group/MDR1 negative group in PEOC, breast cancer and colorectal cancer $(P<0.05)$. The levels of different tumor marker in different groups were shown in Table II.

MDR1 expression and tumor marker level in common malignancies

Receiver operating characteristic (ROC) curve of the predicted probability of logistic regression were constructed, and relative sensitivity and specificity of MDR1 expression and tumor markers were calculated. Area under ROC curves of MDR1 expression and tumor markers were shown in Table III and Figure 3. And partial correlations were used to analyze the correlation between MDR1 expression and tumor markers, we found a significant correlation between MDR1 expression and tumor markers, regardless of whether recurrence was involved in PEOC and breast cancer. The correlation between the level of MDR1 expression and tumor markers were shown in Table IV.

Table I. Relationship between the expression of MDR1 and clinicopathologic paramenters in common malignancies.

\begin{tabular}{lllll}
\hline Tumors & Characteristics & n & Expression & P \\
\hline NSCLC & & & & \\
& Differentiation & & & \\
& Well & 26 & $0.56 \pm 0.20$ & \\
& Moderately +poorly & 21 & $0.36 \pm 0.24$ & 0.004 \\
& Lymphatic metastasis & & & \\
& positive & 30 & $0.59 \pm 0.21$ & \\
& negative & 17 & $0.31 \pm 0.20$ & 0.001
\end{tabular}

Colorectal cancer

$\begin{array}{llll}\text { Duck stage } & & & \\ \text { A+B } & 26 & 0.40 \pm 0.22 & \\ \text { C+D } & 25 & 0.56 \pm 0.31 & 0.047 \\ \text { Lymphatic metastasis } & & & \\ \text { positive } & 34 & 0.55 \pm 0.27 & \\ \text { negative } & 17 & 0.34 \pm 0.24 & 0.01\end{array}$

PEOC

$\begin{array}{llll}\text { Differentiation } & & & \\ \text { Well+moderately } & 32 & 0.51 \pm 0.26 & 0.03 \\ \text { Poorly } & 25 & 0.35 \pm 0.28 & \end{array}$

Gastric cancer

$\begin{array}{lll}\text { Differentiation } & & \\ \text { Well+moderately } & 28 & 0.66 \pm 0.24 \\ \text { Poorly } & 25 & 0.46 \pm 0.26\end{array}$
0.007

Breast cancer

$\begin{array}{llll}\text { Lymphatic metastasis } & & & \\ \text { positive } & 18 & 0.67 \pm 0.21 & \\ \text { negative } & 30 & 0.29 \pm 0.21 & 0.001 \\ \begin{array}{l}\text { Tumor metastasis } \\ \text { Yes }\end{array} & 23 & 0.63 \pm 0.23 & \\ \text { No } & 25 & 0.24 \pm 0.17 & 0.001\end{array}$

Note: PEOC, primary epithelial ovarian cancer; NSCLC, non-small cell lung cancer. MDR1, multidrug resistance 1. 

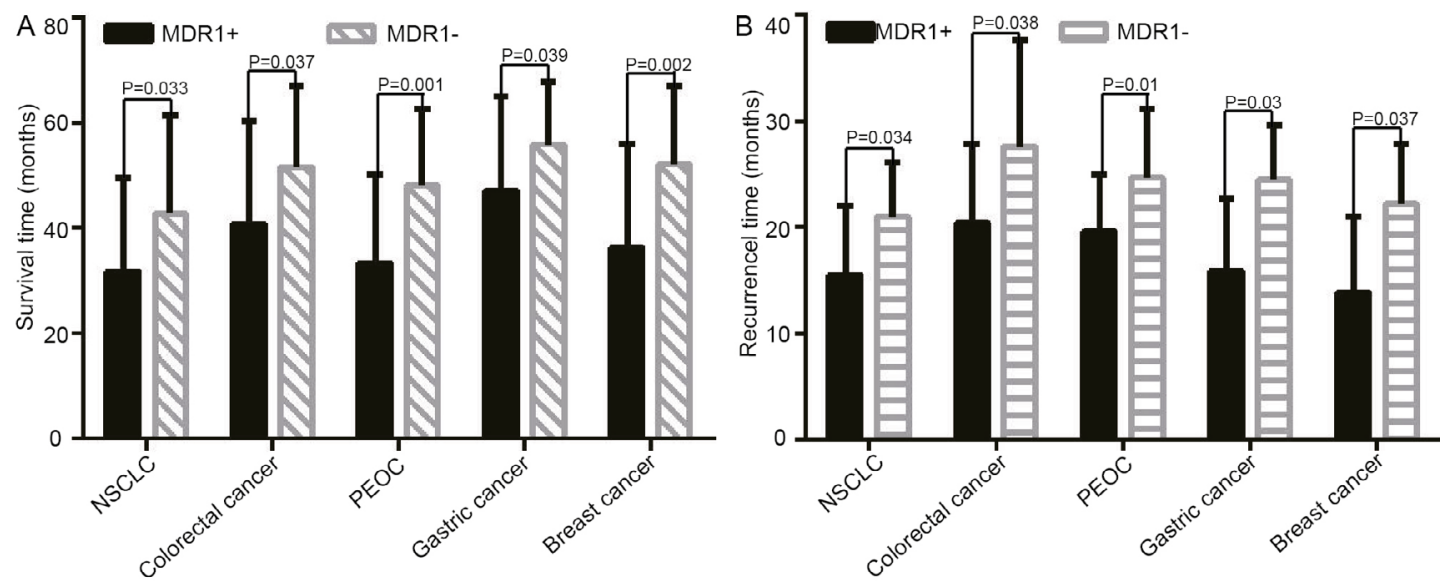

Fig. 2. Recurrence time and survival time in different groups. PEOC: primary epithelial ovarian cancer; NSCLC: non-small cell lung cancer; MDR1: multidrug resistance 1; MDR1+: MDR1 positive group; MDR1-: MDR1 negative group.

Table II. The levels of different tumor markers in different groups.

\begin{tabular}{llllllll}
\hline & & Recurrent & Non-recurrent & P & MDR1 + & MDR1 - & P \\
\hline NSCLS & CYFRA21-1 & & & & & & \\
& pre-operation & $4.89 \pm 1.39$ & $4.73 \pm 1.01$ & 0.66 & $5.06 \pm 1.22$ & $4.52 \pm 1.19$ & 0.14 \\
& post-operation & $3.82 \pm 1.17$ & $3.17 \pm 1.01$ & 0.05 & $3.64 \pm 1.17$ & $3.35 \pm 1.10$ & 0.389 \\
Colorectal cancer & CEA & & & & & \\
& pre-operation & $13.08 \pm 4.79$ & $12.35 \pm 4.69$ & 0.59 & $13.06 \pm 5.39$ & $12.43 \pm 3.89$ & 0.63 \\
& post-operation & $8.36 \pm 2.10$ & $6.17 \pm 2.24$ & 0.001 & $8.05 \pm 2.41$ & $6.71 \pm 2.23$ & 0.046 \\
PEOC & CA125 & & & & & \\
& pre-operation & $31.09 \pm 9.46$ & $27.42 \pm 5.76$ & 0.164 & $31.58 \pm 8.09$ & $28.27 \pm 9.35$ & 0.17 \\
Gastric cancer & post-operation & $15.04 \pm 4.25$ & $11.07 \pm 3.76$ & 0.002 & $15.84 \pm 4.48$ & $11.63 \pm 3.16$ & $<0.001$ \\
& CA724 & & & & & \\
& pre-operation & $8.79 \pm 3.01$ & $9.68 \pm 2.84$ & 0.31 & $9.18 \pm 2.76$ & $9.70 \pm 3.09$ & 0.523 \\
Breast cancer & post-operation & $3.61 \pm 1.79$ & $4.71 \pm 1.83$ & 0.052 & $4.80 \pm 2.02$ & $3.87 \pm 1.57$ & 0.072 \\
& CA153 & & & & & \\
& pre-operation & $32.76 \pm 5.96$ & $29.18 \pm 5.66$ & 0.055 & $31.37 \pm 4.77$ & $28.62 \pm 7.04$ & 0.114 \\
& post-operation & $17.72 \pm 5.68$ & $14.02 \pm 4.12$ & 0.015 & $17.57 \pm 3.96$ & $13.34 \pm 4.75$ & 0.002 \\
\hline
\end{tabular}

Note: PEOC, primary epithelial ovarian cancer; NSCLC, non-small cell lung cancer. MDR1, multidrug resistance 1.
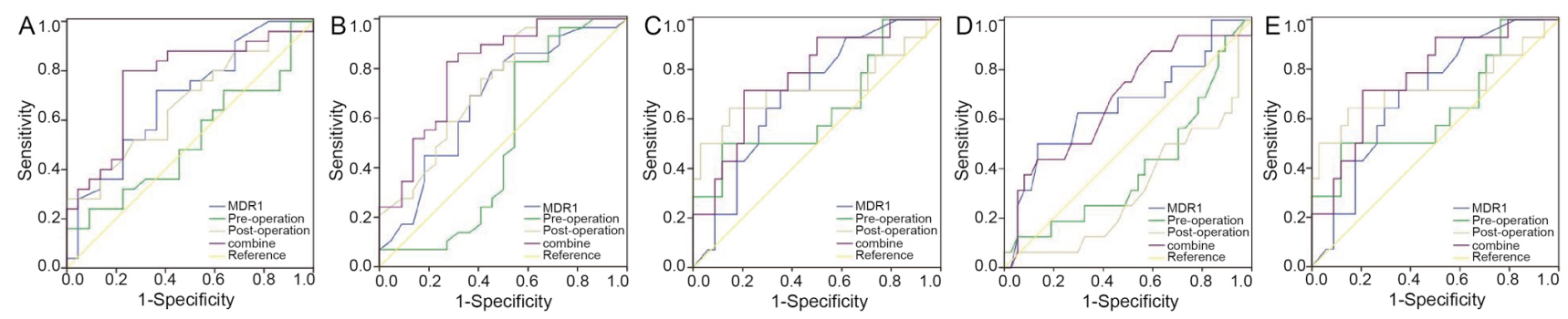

Fig. 3. The ROC analysis of MDR1 expression and tumor markers in the prognosis of common malignant tumor. 

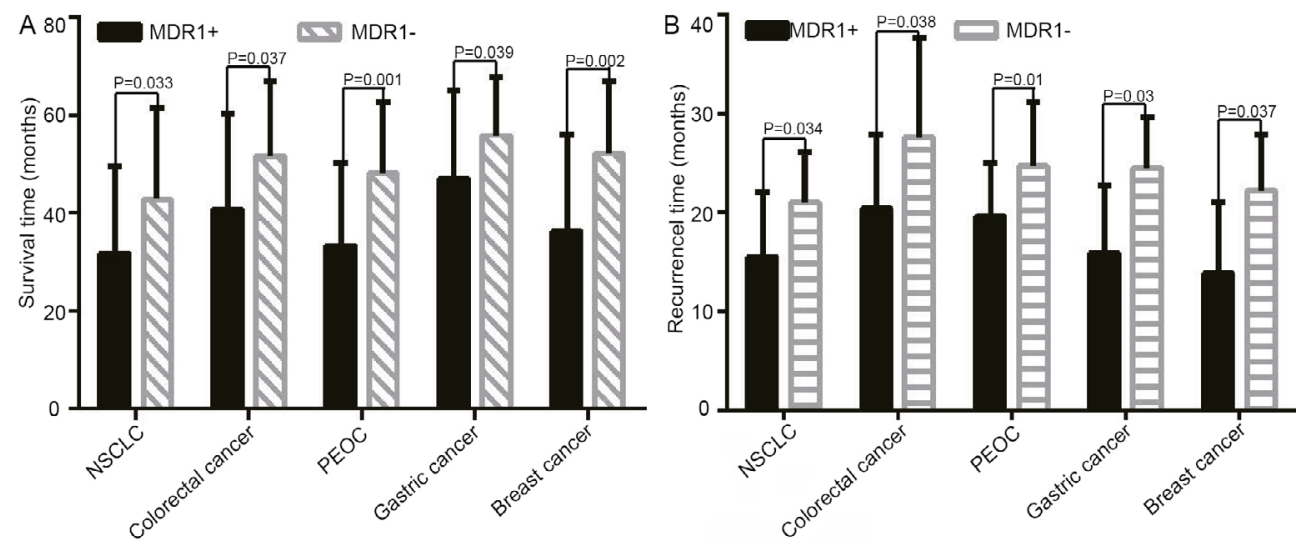

Fig. 4. Survival curves of MDR1 mRNA-positive and negative patients. A: non-small cell lung cancer; B: colorectal cancer; C: primary epithelial ovarian cancer; D: gastric cancer; E:breast cancer.

Table III. Area under ROC curves of MDR1 and tumor markers.

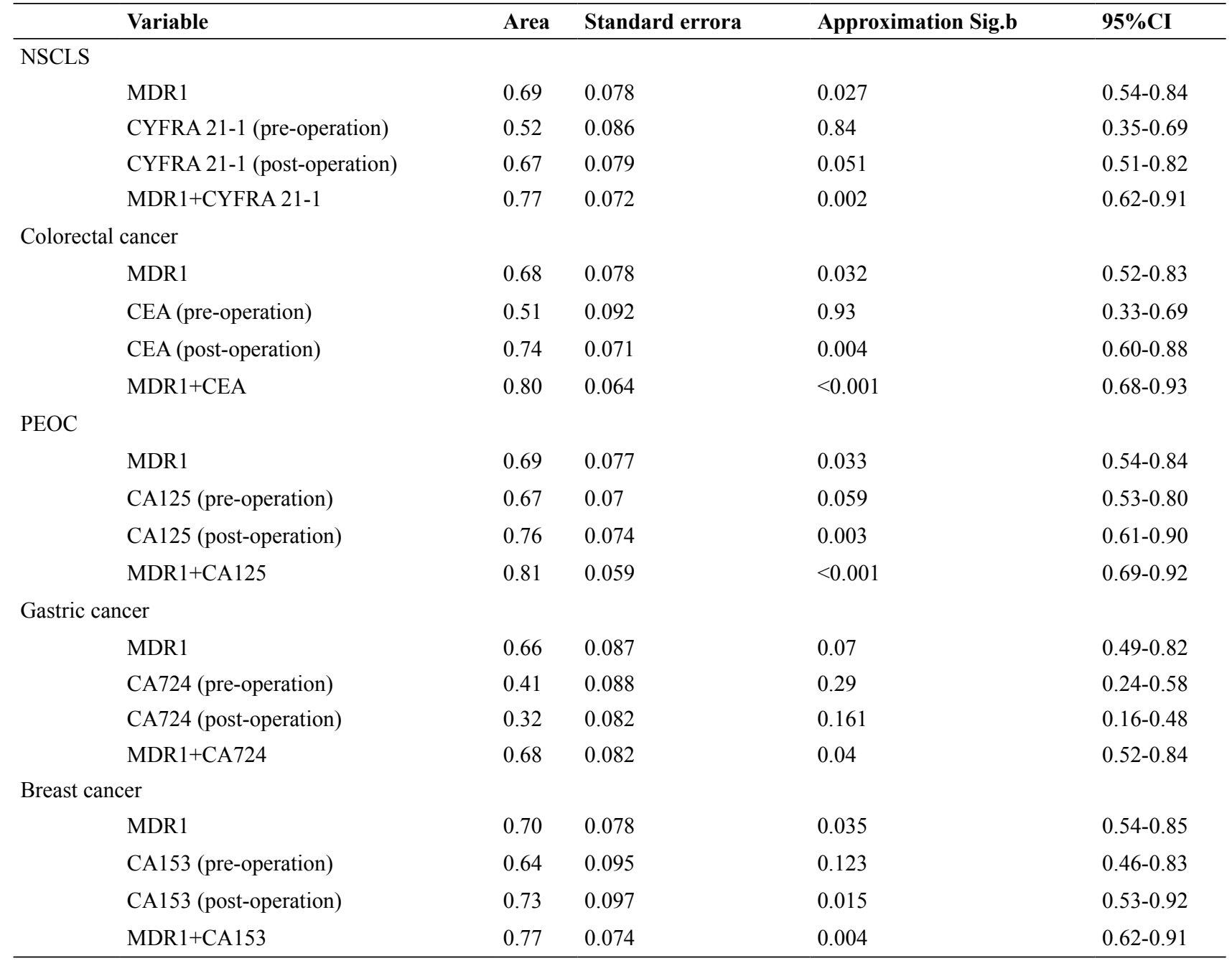

Note: PEOC, primary epithelial ovarian cancer; NSCLC, non-small cell lung cancer. 
Prognostic value of MDR1 expression in common malignancies

Overall survival analysis in the all patients with different types common malignancies based on the relative expression level of MDR1 was carried out by logrank test with Kaplan-Meier method. And the statistical results showed that there were significant differences in the survival curve between the positive group and the negative group based on MDR1 relative expression level in common malignant tumor cases (Fig. 4). Multivariate logistic regression analysis and Univariate logistic regression analysis indicated that high MDR1 expression was significantly associated with common malignancies patients prognosis and was an independent risk factor for malignancies prognosis (Table V).

Table IV. The correlation between the level of MDR1 expression and tumor markers.

\begin{tabular}{llllllllllll}
\hline & CYFRA21-1 & & CEA & & CA125 & & CA724 & & CA153 \\
\hline & & r & P & r & P & r & P & r & P & r & P \\
\hline 1 & MDR1 & 0.052 & 0.73 & 0.22 & 0.19 & 0.42 & 0.001 & 0.018 & 0.9 & 0.38 & 0.007 \\
2 & MDR1 & 0.039 & 0.80 & 0.089 & 0.54 & 0.33 & 0.013 & 0.037 & 0.80 & 0.32 & 0.03 \\
\hline
\end{tabular}

Note: r: Partial correlation coefficient; 1: including recurrent factor; 2: no-including recurrent factor; MDR1, multidrug resistance 1.

Table V. Cox regression analyses for MDR1 in common malignancies.

\begin{tabular}{|c|c|c|c|c|c|c|c|}
\hline \multirow[t]{2}{*}{ Tumors } & \multirow[t]{2}{*}{ Characteristics } & \multicolumn{3}{|c|}{ Univariate analysis } & \multicolumn{3}{|c|}{ Multivariate analysis } \\
\hline & & B & $\operatorname{Exp}(B)$ & $\mathbf{P}$ & B & $\operatorname{Exp}(B)$ & $\mathbf{P}$ \\
\hline \multicolumn{8}{|l|}{ NSCLC } \\
\hline & Lymph node status & & & & & & \\
\hline & metastasis vs No-metastasis & 4.19 & 0.015 & 0.024 & & & \\
\hline & MDR1 & & & & & & \\
\hline & positive vs negative & 3.75 & 42.65 & 0.005 & 1.49 & 4.46 & 0.003 \\
\hline \multicolumn{8}{|c|}{ Colorectal cancer } \\
\hline & Duck Stage & & & & & & \\
\hline & $\mathrm{A}+\mathrm{B}$ VS $\mathrm{C}+\mathrm{D}$ & 1.77 & 5.84 & 0.047 & & & \\
\hline & MDR1 & & & & & & \\
\hline & positive vs negative & 2.00 & 7.36 & 0.028 & 4.02 & 5.47 & 0.043 \\
\hline \multicolumn{8}{|l|}{ PEOC } \\
\hline & Differentiation & & & & & & \\
\hline & Well+moderately VS Poorly & 1.70 & 0.18 & 0.022 & & & \\
\hline & MDR1 & & & & & & \\
\hline & positive vs negative & 2.38 & 0.092 & 0.002 & 1.27 & 3.58 & 0.001 \\
\hline \multicolumn{8}{|c|}{ Gastric cancer } \\
\hline & MDR1 & & & & & & \\
\hline & positive vs negative & 2.20 & 9.00 & 0.013 & 4.41 & 82.40 & 0.039 \\
\hline \multicolumn{8}{|c|}{ Breast cancer } \\
\hline & MDR1 & & & & & & \\
\hline & positive vs negative & 4.09 & 60.00 & 0.002 & 1.67 & 5.30 & 0.005 \\
\hline & Lymph node status & & & & & & \\
\hline & metastasis vs No-metastasis & 1.08 & 2.96 & 0.04 & & & \\
\hline
\end{tabular}

Note: PEOC, primary epithelial ovarian cancer; NSCLC, non-small cell lung cancer; B, regression coeffcient; Exp (B), odds ratio. 


\section{DISCUSSION}

MDR is the manifestation of the declining sensitivity of tumor cells to drugs with different structures and mechanisms (Hu et al., 2017). Among the numerous mechanisms used to produce MDR, the most important one is the protein transport mechanism in cell and nuclear membranes, which are the means through which chemotherapeutic drugs and harmful toxins are transported out of cells. Different types of antitumor drugs can be expelled out of the cell (Levatic et al., 2013; Loo et al., 2013). P-gp is encoded by MDR1 and plays a particularly important role as a drug carrier in different MDR mechanisms. High expression of resistance genes is the main cause of tumor cell resistance to chemotherapy (Chufan et al., 2015; Hou et al., 2008). The drug resistance gene may be an important biomarker in predicting and improving tumor prognosis.

In this study, we found that relative MDR1 gene expression levels in common malignancies were significantly higher than those in adjacent and noncancerous tissues. None of the patients underwent preoperative radiotherapy or chemotherapy, which suggests that primary drug resistance is associated with common malignancies. MDR1 expression was also associated with clinic pathological features that may represent tumor prognosis, such as differentiation degree or lymphatic metastasis. Thus, high MDR1 expression may be involved in the prognosis of common malignant tumors. The prognosis and recurrence of tumors may be closely related to the high expression of MDR1 (Sun et al., 2016).

We divided all of the cases into a positive-MDR1 expression group and a negative-MDR1 expression group according to expression level and studied the recurrence and survival times of patients in both groups. Statistical analysis revealed that, in several common malignancies, the recurrence and survival times of positive-MDR1 expression patients were shorter than those of negativeexpression patients. Survival curve analysis demonstrated that MDR1 expression in common malignant tumor patients of the negative-group survival curves was higher than that in the positive-group curves. This phenomenon has also been observed in cervical cancer (Yang et al., 2017) and liver cancer (Gao et al., 2015), thereby suggesting that high MDR1 expression may be closely related to the prognosis of common malignancies and that high expression level could indicate poor prognosis for common malignant tumor cases.

A large body of evidence shows that drug resistance genes could be used as prognostic indicators of cancer (Janikova et al., 2016; Pan et al., 2016). In the present study, we investigated the prognostic value of the relative expression of the MDR1 gene in common malignant tumor cases. Multivariate and univariate logistic regression analyses indicated that high relative expression level of the MDR1 gene could be an independent prognostic risk factor for malignant tumor cases. The expression level of various resistance genes can be used as prognostic markers for cervical and liver cancer (Kim et al., 2016; Dufour et al., 2015). However, the importance of these relative expression level has rarely been reported in clinical applications. Therefore, in future studies (including in vitro and in vivo), increasing the sample size is necessary to confirm the prognostic value of the relative expression the MDR1 gene in common malignant tumor cases.

As the primary treatment for most malignant tumors, surgery can achieve a certain therapeutic effect, the level of tumor markers after surgery was significantly reduced, and tumor markers can be maintained at a normal level with postoperative chemotherapy. However, multidrug resistance can affect the sensitivity of tumor cells to chemotherapeutic agents and lead to tumor recurrence. Studies have shown that CA125, CA153 and CEA may be useful in predicting the recurrence of ovarian cancer (Guo et al., 2017), breast cancer (Li et al., 2017) and colorectal cancer (Gao et al., 2018). When the value of CA125 is higher than $10 \mathrm{U} / \mathrm{ml}$ and continuously increased, indicates a relative risk of recurrence and need to be vigilant (Guo et al., 2017). Nicholson BD (Nicholson et al., 2015) recommend monitoring for colorectal cancer recurrence with more than one diagnostic modality but applying the highest CEA cut-off assessed $(10 \mu \mathrm{g} / \mathrm{L})$. In this study, we also studied the value of MDR1 expression and tumor markers in predicting recurrence of malignant tumors. Relative sensitivity and specificity of MDR1 expression and tumor markers were calculated by ROC in this study. The detection of MDR1 expression combined with tumor markers can improve the sensitivity and specificity of predicting postoperative recurrence. After surgery, there was statistically significant difference in the level of tumor markers between the recurrence group/the non-recurrence group and MDR1 positive group/MDR1 negative group in PEOC, breast cancer and colorectal cancer. Besides, we found a significant correlation between MDR1 expression and tumor markers, regardless of whether recurrence was involved in PEOC and breast cancer by partial correlations analysis, which suggested that the expression of MDR1 can indirectly predict the postoperative level of CA125 and CA153. Although CA724 and CYFBA21-1 can be used for non-specific diagnosis of gastric cancer ( $\mathrm{Li}$ et al.,2013) and non-small cell lung cancer (NSCLC) (Yu et al., 2017), they need to be further studied as tumor markers for predicting postoperative recurrence. 


\section{CONCLUSION}

In conclusion, high MDR1 expression is related to aggressive clinical characteristics in common malignant tumor cases. Thus, MDR1 may be a high-risk factor of prognosis for common malignancies.

\section{ACKNOWLEDGEMENT}

This study were funded by Taihe hospital projects (No. 2016JZ26, No. 2017JJXM044 and N0. 2014JJXM019).

\section{Statement of conflict of interest}

There is no conflict of interest.

\section{REFERENCES}

Abraham, J., Salama, N.N. and Azab, A.K., 2015. The role of P-glycoprotein in drug resistance in multiple myeloma. Leuk. Lymph., 56: 26-33. https://doi.org/1 0.3109/10428194.2014.907890

Chen, W., Zheng, R. and Baade, P.D., 2016. Cancer statistics in China, 2015. CA. Cancer J. Clin., 66: 115-132. https://doi.org/10.3322/caac.21338

Chufan, E.E., Sim, H.M. and Ambudkar, S.V., 2015. Molecular basis of the polyspecificity of P-glycoprotein (ABCB1): Recent biochemical and structural studies. Adv. Cancer Res., 125: 7196. https://doi.org/10.1016/bs.acr.2014.10.003

Dufour, R., Daumar, P. and Mounetou, E., 2015. BCRP and P-gp relay overexpression in triple negative basal-like breast cancer cell line: a prospective role in resistance to Olaparib. Sci. Rep., 5: 12670. https:// doi.org/10.1038/srep12670

Gao, B., Yang, F.M. and Yu, Z.T., 2015. Relationship between the expression of MDR1 in hepatocellular cancer and its biological behaviors. Int. J. clin. exp. Pathol., 8: 6995-7001.

Gao, Y., Wang, J. and Zhou, Y., 2018. Evaluation of serum CEA, CA19-9, CA72-4, CA125 and ferritin as diagnostic markers and factors of clinical parameters for colorectal Cancer. Sci. Rep., 8: 2732. https://doi.org/10.1038/s41598018-21048-y

Guo, N. and Peng, Z., 2017. Does serum CA125 have clinical value for follow-up monitoring of postoperative patients with epithelial ovarian cancer? Results of a 12-year study. J. Ovarian Res., 10: 14. https://doi.org/10.1186/s13048-017-0310-y

Ge, C., Cao, B. and Feng, D., 2017. The downregulation of SLC7A11 enhances ROS induced
P-gp over-expression and drug resistance in MCF7 breast cancer cells. Sci. Rep., 7: 3791. https://doi. org/10.1038/s41598-017-03881-9

Hou, X.L, Takahashi, K. and Tanaka, K., 2008. Curcuma drugs and curcumin regulate the expression and function of P-gp in Caco-2 cells in completely opposite ways. Int. J. Pharm., 358: 224-229. https://doi.org/10.1016/j.ijpharm.2008.03.010

Hu, Y., Qin. X. and Cao, H., 2017. Reversa'l effects of local anesthetics on P-glycoprotein-mediated cancer multidrug resistance. Anticancer Drugs, 28: 243-249. https://doi.org/10.1097/CAD.0000000000000455

Janikova, M.,Zizkova, V. and Skarda, J., 2016. Prognostic significance of miR-23b in combination with P-gp, MRP and LRP/MVP expression in non-small cell lung cancer. Neoplasma, 63: 576-587. https://doi. org/10.4149/neo_2016_411

Joshi, P., Vishwakarma, R.A., and Bharate, S.B., 2017. Natural alkaloids as P-gp inhibitors for multidrug resistance reversal in cancer. Eur. J. med. Chem., 138: 273-292. https://doi.org/10.1016/j. ejmech.2017.06.047

Li, F., Li, S. and Wei, L., 2013. The correlation between pre-operative serum tumor markers and lymph node metastasis in gastric cancer patients undergoing curative treatment. Biomarkers, 18: 632-637. https:// doi.org/10.3109/1354750X.2013.840800

Kim, J.W., Park, Y. and Roh, J.L. 2016. Prognostic value of glucosylceramide synthase and P-glycoprotein expression in oral cavity cancer. Int. J. clin. Oncol., 21: 883-889. https://doi.org/10.1007/s10147-0160973-1

Li, L., Gao, Q. and Xu, G., 2017. Postoperative recurrence analysis of breast cancer patients based on clinical serum markers using discriminant methods. Cancer Biomark, 19: 403-409. https://doi.org/10.3233/ CBM-160322

Liu, Z., Duan, Z.J. and Chang, J.Y., 2104. Sinomenine sensitizes multidrug-resistant colon cancer cells (Caco-2) to doxorubicin by downregulation of MDR-1 expression. PLoS One, 9: e98560. https:// doi.org/10.1371/journal.pone.0098560

Levatić, J., Ćurak. J. and Kralj, M., 2013. Accurate models for P-gp drug recognition induced from a cancer cell line cytotoxicity screen. J. med. Chem., 56: $5691-5708$. https://doi.org/10.1021/jm400328s

Loo, T.W., Bartlett, M.C. and Clarke, D.M., 2013. Human P-glycoprotein contains a greasy ball-and-socket joint at the second transmission interface. J. biol. Chem., 288: 20326-20333. https://doi.org/10.1074/ jbc.M113.484550 
Mieszala, K., Rudewicz, M. and Gomulkiewicz, A., 2018. Expression of genes and proteins of multidrug resistance in gastric cancer cells treated with resveratrol. Oncol. Lett., 15: 5825-5832. https://doi. org/10.3892/ol.2018.8022

Nicholson, B.D., Shinkins, B. and Pathiraja, I., 2015. Blood CEA levels for detecting recurrent colorectal cancer. Cochrane Database Syst. Rev., 10: CD011134. https://doi.org/10.1002/14651858. CD011134.pub2

Pan, S.T., Li, Z.L. and He, Z.X., 2016. Molecular mechanisms for tumour resistance to chemotherapy. Clin. exp. Pharmacol. Physiol., 43: 723-737. https:// doi.org/10.1111/1440-1681.12581

Sun, S., Cai, J. and Yang, Q., 2016. Prognostic value and implication for chemotherapy treatment of ABCB1 in epithelial ovarian cancer: A metaanalysis. PLoS One, 11: e0166058. https://doi. org/10.1371/journal.pone.0166058

Yan, L.H., Wei. W.Y. and Cao, W.L., 2014. Overexpression of CDX2 in gastric cancer cells promotes the development of multidrug resistance. Am. J. Cancer Res., 5: 321-332.

Yan, L.H., Wei, W.Y. and Cao. W.L., 2014. Overexpression of E2F1 in human gastric carcinoma is involved in anti-cancer drug resistance. BMC Cancer, 14: 904. https://doi.org/10.1186/1471-2407-14-904

Yang, F., Gao, B. and Li, R., 2017. Expression levels of resistant genes affect cervical cancer prognosis. Mol. Med. Rep., 15: 2802-2806. https://doi.org/10.3892/ mmr.2017.6328

Yu, Z., Zhang, G. and Yang, M., 2017. Systematic review of CYFRA 21-1 as a prognostic indicator and its predictive correlation with clinicopathological features in non-small cell lung cancer: A metaanalysis. Oncotarget, 8: 4043-4050. https://doi. org/10.18632/oncotarget.14022

Zhao, L., Bin, S. and He, H.L., 2018. Sodium butyrate increases P-gp expression in lung cancer by upregulation of STAT3 and mRNA stabilization of ABCB1. Anticancer Drugs, 29: 227-233. https://doi. org/10.1097/CAD.0000000000000588 